\title{
Optimal Portfolios when Volatility can Jump
}

\author{
Nicole Branger ${ }^{\S}$ \\ Christian Schlag $\ddagger$ \\ Eva Schneider $₫$
}

${ }^{\S}$ Department of Business and Economics, University of Southern Denmark, Campusvej 55, DK-5230 Odense M, Denmark, phone: +45 (6550) 3227, fax:+45 6615 8790, e-mail: nbr@sam.sdu.dk

${ }^{\ddagger}$ Finance Department, Goethe University, Mertonstr. 17-21/Uni-Pf 77, D-60054 Frankfurt am Main, Germany, phone: +49 (69) 798 22674, fax: +49 (69) 798 22788, e-mail: schlag@finance.uni-frankfurt.de

『Finance Department, Goethe University, Mertonstr. 17-21/Uni-Pf 77, D-60054 Frankfurt am Main, Germany, phone: +49 (69) 798 25178, fax: +49 (69) 798 22788, e-mail: schneider@finance.uni-frankfurt.de 


\title{
Optimal Portfolios when Volatility can Jump
}

\begin{abstract}
We consider an asset allocation problem in a continuous-time model with stochastic volatility and (possibly correlated) jumps in both, the asset price and its volatility. First, we derive the optimal portfolio for an investor with constant relative risk aversion. One main finding is that the demand for jump risk now also includes a hedging component, which is not present in models without jumps in volatility. Second, we show in a partial equilibrium framework that the introduction of nonlinear derivative contracts can have a substantial economic value. Third, we analyze the distribution of terminal wealth for an investor who uses the wrong model when making portfolio choices, either by ignoring volatility jumps or by falsely including such jumps although they are not present in the true model. In both cases the terminal wealth distribution exhibits fatter tails than under the correctly specified model, as well as significant default risk. Volatility jumps are thus an important risk factor in portfolio planning.
\end{abstract}

JEL: G12, G13

Keywords: Dynamic asset allocation, jump risk, volatility jumps, stochastic volatility, model mis-specification 


\title{
Optimal Portfolios when Volatility can Jump
}

\author{
Nicole Branger ${ }^{\S} \quad$ Christian Schlag ${ }^{\ddagger} \quad$ Eva Schneider $₫$
}

This version: November 30, 2005

\begin{abstract}
We consider an asset allocation problem in a continuous-time model with stochastic volatility and (possibly correlated) jumps in both the asset price and its volatility. First, we derive the optimal portfolio for an investor with constant relative risk aversion. One main finding is that the demand for jump risk now also includes a hedging component, which is not present in models without jumps in volatility. Second, we show in a partial equilibrium framework that the introduction of nonlinear derivative contracts can have a substantial economic value. Third, we analyze the distribution of terminal wealth for an investor who uses the wrong model when making portfolio choices, either by ignoring volatility jumps or by falsely including such jumps although they are not present in the true model. In both cases the terminal wealth distribution exhibits fatter tails than under the correctly specified model, as well as significant default risk. Volatility jumps are thus an important risk factor in portfolio planning.
\end{abstract}

Keywords: Dynamic asset allocation, jump risk, volatility jumps, stochastic volatility, model mis-specification

JEL: G12, G13

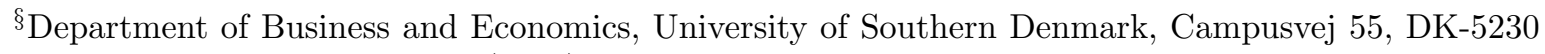
Odense M, Denmark, phone: +45 (6550) 3227, fax:+45 6615 8790, e-mail: nbr@sam.sdu.dk

${ }^{\ddagger}$ Finance Department, Goethe University, Mertonstr. 17-21/Uni-Pf 77, D-60054 Frankfurt am Main, Germany, phone: +49 (69) 798 22674, fax: +49 (69) 798 22788, e-mail: schlag@finance.uni-frankfurt.de

『Finance Department, Goethe University, Mertonstr. 17-21/Uni-Pf 77, D-60054 Frankfurt am Main, Germany, phone: +49 (69) 798 25178, fax: +49 (69) 798 22788, e-mail: schneider@finance.uni-frankfurt.de

Earlier versions of the paper were presented at Vanderbilt University, Universität Göttingen, the European Summer Symposium in Financial Markets in Gerzensee, 2005, at the Annual Meetings of the German Finance Association in Augsburg, 2005, and at Copenhagen Business School. We thank the participants for helpful comments. Special thanks go to Holger Kraft. 


\section{Introduction and Motivation}

The key risk factors considered in option pricing models, besides the diffusive price risk of the underlying asset, are stochastic volatility, jumps in the asset price, and also jumps in volatility. Models that include some or all of these factors were developed by Merton (1976), Heston (1993), Bates (1996), Bakshi, Cao, and Chen (1997), and Duffie, Pan, and Singleton (2000). The importance of jumps in volatility has become apparent in recent studies, which try to explain the time series properties of stock and option prices simultaneously, like Eraker, Johannes, and Polson (2003), or Broadie, Chernov, and Johannes (2005).

In this paper, we analyze the importance and impact of jumps in volatility for portfolio planning. First of all, we investigate the impact of jumps in volatility on the investor's optimal portfolio and on its structure. Second, we assess the utility gain generated by the availability of derivatives in this economy. Third, we analyze the distribution of terminal wealth for an investor who uses the wrong model, namely either one that does not contain volatility jumps although the true model does, or one containing such jumps although they are not part of the true model. Our results show that jumps in volatility are an important risk factor when it comes to portfolio planning. Their inclusion or omission changes the structure of the optimal portfolio. Furthermore, the use of a wrong model that either ignores volatility jumps or wrongly includes them results in economically significant utility losses.

There are important fundamental differences between stochastic volatility and stochastic jumps, as shown in the area of contingent claim pricing by Das and Sundaram (1999) and Carr and $\mathrm{Wu}$ (2002), and concerning option hedges by Branger and Schlag (2004). In an asset allocation context the main papers analyzing the impact of jumps are Liu, Longstaff, and Pan (2003), Liu and Pan (2003) and Dieckmann and Gallmeyer (2004). Whereas Dieckmann and Gallmeyer (2004) consider the allocation of diffusive and jump risks between heterogeneous agents in a pure exchange economy, Liu, Longstaff, and Pan (2003) and Liu and Pan (2003) are operating in a partial equilibrium framework.

Our analysis ties up some loose ends in the literature on asset allocation in continuoustime models with jumps. We consider the portfolio planning problem in a very general setup with stochastic volatility, jumps in the stock price, and, in particular, jumps in volatility. Thereby, we extend the comparison of diffusion risk and jump risk in Liu, Longstaff, and Pan (2003) to the more realistic case when derivatives are actually available to the investor. By considering a model that includes jumps in volatility, we also extend the framework in Liu and Pan (2003) who study the benefits from trading derivatives in a model without jumps in volatility.

Our framework represents a significant generalization of both of these papers. We solve the model in closed form for the case of imperfectly correlated jumps in the stock price and in volatility. Our model can capture jumps in the stock price only, jumps in volatility only, and simultaneous jumps in both processes. We can thus analyze structural differences between these kinds of jumps. For the numerical analysis, we restrict the model 
to the more simple case where jump sizes are deterministic and where both the stock and the volatility jump simultaneously. This allows us to focus on the key aspects of our model and it also allows for an easy comparison with the results of Liu, Longstaff, and Pan (2003) and Liu and Pan (2003).

First, we derive the optimal portfolio of an investor with constant relative risk aversion and analyze its structure. To concentrate on the impact of jumps, we assume that the market is complete, i.e. enough derivatives are traded. In the spirit of Merton (1971) we separate the overall demand for a risk factor into a speculative component, which represents the investor's desire to earn the associated risk premium, and the hedging component, which serves to protect the investor against unfavorable changes in the investment opportunity set. Our main finding is that, with jumps in volatility, the optimal demand for jump risk now also contains a hedging component, which is not present in the Liu and Pan (2003) economy without jumps in volatility. Intuitively, the hedging demand can be explained by the desire of the investor to hedge against future unfavorable changes in volatility. When jumps have an impact on volatility, part of this hedging can be achieved by trading jump risk, while in the case without jumps in volatility, all the hedging has to be done by trading diffusion risk. The omission of jump risk in volatility will thus lead to an overestimation of the hedging demand for diffusion risk.

Second, we determine the economic value of trading in derivatives. Derivatives are a vehicle to achieve the optimal exposure to the fundamental risk factors in an economy, i.e. to diffusions and jump components. The introduction of derivatives thus always increases the investor's utility in a partial equilibrium model, where we assume the market prices of existing assets to remain unchanged. We measure the economic gain due to derivatives by the annualized percentage increase in certainty equivalent wealth, which can be thought of as a kind of an additional interest rate. Our results show that this gain is economically significant.

Third, we analyze the impact of model mis-specification on the portfolio planning problem. Given that the true model is not known and has to be estimated, we assume that the investor either wrongly uses a model without jumps in volatility which is calibrated to market data, or that he includes volatility jumps in the asset allocation although they are not present in the true data generating process. In both situations with model misspecification, we show that the distribution of terminal wealth exhibits more mass in both, the left and the right tail. In particular, the risk of obtaining a very low terminal wealth and thus of bankruptcy increases significantly. Our results show that neither the use of a too parsimonious nor of a too sophisticated model provides a simple robust hedge with respect to model risk.

Our paper is mainly related to Liu, Longstaff, and Pan (2003) andLiu and Pan (2003). In a stochastic volatility model with deterministic jumps in the stock price and no jumps in volatility, Liu and Pan (2003) derive closed-form solutions for the optimal portfolio composition in the case of a CRRA utility function. They show that derivatives can be used to achieve the desired exposure to each risk factor. The reason is that such nonlinear contracts can be employed to disentangle jump risk and diffusion risk which, in the case of the stock, are only available as one 'package'. Furthermore, derivatives allow to trade 
volatility risk. Liu, Longstaff, and Pan (2003) propose a more general framework for the dynamics of the state variables by including volatility jumps, but restrict the set of traded assets to the stock and the money market account. They find that compared to the case of no stock price jumps, the investor reduces the position in the risky asset even when jumps are upward. In the most general case with jumps in both price and volatility the position in the risky asset can increase or decrease compared to the no jump case, since jumps in volatility enable at least a partial hedging of jumps in the stock price.

Another paper related to ours is the study by Daglish (2002). He considers the same fundamental model as Liu, Longstaff, and Pan (2003), but allows for stochastic jumps instead of assuming deterministic jump sizes. With the stock and the money market account only, the market is incomplete. There are no closed-form solutions and the analysis has to be done numerically. For the case of log-normally distributed jumps, his results confirm those of Liu, Longstaff, and Pan (2003). Since the market is incomplete, it is thus no longer possible to work in terms of risk factors, but one has to consider demands for exogenously given specific assets instead. In contrast, we retain a complete market by assuming that a sufficiently large number of derivatives is traded to span even stochastic jumps which are drawn from a discrete distribution. This allows us to focus on the structural impact of jumps.

$\mathrm{Wu}$ (2003) investigates a jump-diffusion model, in which the diffusion risk premium follows an Ornstein-Uhlenbeck process. With the stock and the money market account as traded assets, he finds that there is interaction between return predictability and the impact of jump risk. He also provides a detailed analysis of the impact of jumps as the source of non-normality on portfolio decisions in one-period and multi-period models. Ilhan, Jonsson, and Sircar (2004) compute asymptotic approximations to the optimal derivative holdings based on an indifference argument in a world with purely diffusive stochastic volatility. Finally, Das and Uppal (2004) analyze the impact of systemic jump risk on the optimal portfolio and on the utility of an investor with CRRA, as well as the utility loss when this risk is ignored. In their paper, systemic jump risk is modeled as a common jump across all asset prices but does not comprehend jumps in volatility.

The remainder of the paper is structured as follows. In Section 2 we present the model. Section 3 contains the solution to the portfolio planning problem and its economic interpretation. Section 4 provides a numerical example for the impact of jumps in volatility. The economic value of derivatives in the context of our model is discussed in Section 5 and model mis-specification is analyzed in Section 6. Section 7 concludes. 


\section{Model Setup}

The dynamics of the stock price $S$ and the instantaneous variance $V$ under the true measure $P$ are given by the following system of stochastic differential equations:

$$
\begin{aligned}
d S_{t}= & \mu_{t} S_{t} d t+\sqrt{V_{t}} S_{t} d B_{t}^{(1)}+S_{t-}\left(\sum_{j, k} x^{(j)} d N_{t}^{(j, k)}-E^{P}[X] \lambda^{P} V_{t} d t\right) \\
d V_{t}= & \kappa^{P}\left(\bar{v}^{P}-V_{t}\right) d t+\sigma_{V} \sqrt{V_{t}}\left(\rho d B_{t}^{(1)}+\sqrt{1-\rho^{2}} d B_{t}^{(2)}\right) \\
& +\left(\sum_{j, k} y^{(k)} d N_{t}^{(j, k)}-E^{P}[Y] \lambda^{P} V_{t} d t\right) .
\end{aligned}
$$

The asset price and variance are driven by the independent Brownian motions $B^{(1)}$ and $B^{(2)}$ and by $M \equiv J \cdot K$ independent Poisson processes $N_{t}^{(j, k)}$, each with (stochastic) intensity $\lambda^{P} V_{t} p_{j k}$. The physical probability that a jump occurs over the next interval of length $d t$ at all is equal to $\lambda^{P} V_{t} d t$, and given that a jump has occurred, the random jump sizes $(X, Y)$ have realizations $\left(x^{(j)}, y^{(k)}\right)$ with probabilities $p_{j k}$. In general, the jump sizes for both, the asset price and the variance, are stochastic. We assume that these jump sizes are discrete random variables with possible realizations $x^{(j)}, j=1, \ldots, J$ for the stock and $y^{(k)}, k=1, \ldots, K$ for the variance. The variance jumps have to be restricted to $y^{(k)} \geq 0$ for $k=1, \ldots, K$, in order to avoid negative values for $V \cdot \bar{v}^{P}$ is the long-run mean of the variance.

This setup allows us to model three different kinds of jumps: jumps in the stock price only, jumps in the variance only, and simultaneous jumps in both processes. We set $x^{(1)}=0$ and $y^{(1)}=0$. Table 1 summarizes the structure. Jumps in the stock price only can be described by pairs $\left(x^{(j)}, y^{(1)}\right)=\left(x^{(j)}, 0\right)$ for $j \geq 2$. These jumps have an individual intensity under the $P$-measure equal to $\lambda^{P} V_{t} p_{j 1}$, so that the intensity for a pure stock price jump is given by $\lambda^{P} V_{t} \sum_{j=2}^{J} p_{j 1}$. Analogously, pure variance jumps are represented by pairs $\left(x^{(1)}, y^{(k)}\right)=\left(0, y^{(k)}\right)$ for $k \geq 2$. The $P$-intensity for such a jump is given by $\lambda^{P} V_{t} \sum_{k=2}^{K} p_{1 k}$. The rest of the probability mass is distributed over all possible realizations of simultaneous jumps $\left(x^{(j)}, y^{(k)}\right), j, k \geq 2$ in the stock price and in $V$. The correlation structure of price and variance jumps can be generated by an appropriate specification of the joint probabilities. Note that the event $\left(x^{(1)}, y^{(1)}\right)=(0,0)$ is not considered in the jump size distribution (i.e. is assigned a zero probability), since it obviously represents the case of no jump at all. Even if we do not restrict the joint distribution of $X$ and $Y$ besides $y^{(k)} \geq 0$ for all $k=1, \ldots, K$, the most natural and empirically well-supported structure would be one with a negative jump size for prices (at least on average) and upward jumps in volatility. A typical jump event would thus decrease prices and simultaneously increase volatility, which can be regarded as an increase in uncertainty after a market crash.

The interest rate $r$ is constant. The market prices of risk in our model are not unique, but have to be given exogenously. Following Liu and Pan (2003), we specify the pricing 
kernel $\xi$ via the stochastic differential equation

$$
\begin{aligned}
d \xi_{t}= & -\xi_{t}\left(r d t+\eta^{B 1} \sqrt{V_{t}} d B_{t}^{(1)}+\eta^{B 2} \sqrt{V_{t}} d B_{t}^{(2)}\right) \\
& +\xi_{t-}\left\{\sum_{j, k}\left(\frac{\lambda^{Q} q_{j k}}{\lambda^{P} p_{j k}}-1\right) d N_{t}^{(j, k)}-\left(\frac{\lambda^{Q}}{\lambda^{P}}-1\right) \lambda^{P} V_{t} d t\right\} .
\end{aligned}
$$

The market price of risk $\eta^{B 1} V_{t}$ represents the compensation per unit of $\sqrt{V_{t}} d B_{t}^{(1)}$, while $\eta^{B 2} V_{t}$ is the expected reward for bearing one unit of $\sqrt{V_{t}} d B_{t}^{(2)}$. The compensation for an exposure of $+\alpha$ to a jump of size $\left(x^{(j)}, y^{(k)}\right)$ (i.e. for an increase in wealth of $\alpha \cdot 100 \%$ if such a jumps occurs) is given by $\alpha \cdot\left[p_{j k} \lambda^{P}-q_{j k} \lambda^{Q}\right] V_{t}$.

From these specifications we obtain the following dynamics under the risk-neutral measure $Q$ :

$$
\begin{aligned}
d S_{t}= & r S_{t} d t+\sqrt{V_{t}} S_{t} d \widetilde{B}_{t}^{(1)}+S_{t-}\left(\sum_{j, k} x^{(j)} d N_{t}^{(j, k)}-E^{Q}[X] \lambda^{Q} V_{t} d t\right) \\
d V_{t}= & \kappa^{Q}\left(\bar{v}^{Q}-V_{t}\right) d t+\sigma_{V} \sqrt{V_{t}}\left(\rho d \widetilde{B}_{t}^{(1)}+\sqrt{1-\rho^{2}} d \widetilde{B}_{t}^{(2)}\right) \\
& +\left(\sum_{j, k} y^{(k)} d N_{t}^{(j, k)}-E^{Q}[Y] \lambda^{Q} V_{t} d t\right),
\end{aligned}
$$

where the intensity of the Poisson process $d N_{t}^{(j, k)}$ is now $\lambda^{Q} V_{t} q_{j k}$. In the general case, both, the jump intensity and the jump size distribution, are different under $P$ and $Q$. The parameters of the volatility process under the measures $P$ and $Q$ are related via

$\kappa^{Q}\left(\bar{v}^{Q}-V_{t}\right)-E^{Q}[Y] \lambda^{Q} V_{t}=\kappa^{P}\left(\bar{v}^{P}-V_{t}\right)-E^{P}[Y] \lambda^{P} V_{t}-\sigma_{V}\left(\rho \eta^{B 1}+\sqrt{1-\rho^{2}} \eta^{B 2}\right) V_{t}$,

so that

$$
\begin{aligned}
\kappa^{Q} & =\kappa^{P}+\sigma_{V}\left(\rho \eta^{B 1}+\sqrt{1-\rho^{2}} \eta^{B 2}\right)+\left(E^{P}[Y] \lambda^{P}-E^{Q}[Y] \lambda^{Q}\right) \\
\kappa^{Q} \bar{v}^{Q} & =\kappa^{P} \bar{v}^{P}
\end{aligned}
$$

The expected excess return on equity is given by

$$
\mu_{t}-r=\left(\eta^{B 1}+E^{P}[X] \lambda^{P}-E^{Q}[X] \lambda^{Q}\right) V_{t}
$$

It combines the compensation for diffusion risk and the compensation for jump risk and is proportional to the local variance $V_{t}$. In the setup of Liu, Longstaff, and Pan (2003), where only the stock and the money market account are traded, the relative size of these two risk premia does not matter. However, in our model where both risk factors can be traded separately, the decomposition of the equity risk premium into the compensations for these two risk factors becomes important. 
A very attractive feature of the analysis in Liu and Pan (2003) is that the market is complete, so that instead of pre-specifying tradable assets and focusing on the investor's demand for this particular set of assets, one can work with the more general concept of demands for the various risk factors driving the economy. In the setup considered here, the market is complete when the number of non-redundant derivatives is equal to the number of possible stock-volatility jump realizations that occur with positive probability plus one to hedge the stochastic volatility factor. In the general case we thus need $M$ additional traded instruments besides the stock and the money market account. To achieve market completeness with a finite number of derivatives, we have to assume that the jump size distribution is discrete with a finite number of possible values but we cannot assume continuous jump size distributions. However, our setup is rich enough to study the case of stochastic jumps and to analyze possible differences to the case of deterministic jumps only, and additionally, differences between the three types of jumps. Furthermore, we have to assume that enough contingent claims are traded. While this may not be the case for all underlyings, we do think this assumption to be justified when we focus on stock indices e.g.. Here we usually observe a large number of options with sufficient trading volume so that an investor could use them for his asset allocation. The assumption of market completeness allows us to focus on the structural impact and economic importance of jumps in volatility.

We now consider the contingent claims that are traded in our economy. Let $O_{t}^{(i)}=$ $g^{(i)}\left(S_{t}, V_{t}\right)(i=1,2, \ldots, M)$ denote the price of the $i$-th derivative as a function of the state variables. The dynamics of the price follow from Ito and the fundamental partial differential equation, and we obtain

$$
\begin{aligned}
d O_{t}^{(i)}= & r O_{t}^{(i)} d t \\
& +\left(g_{s}^{(i)} S_{t}+\sigma_{V} \rho g_{v}^{(i)}\right)\left(\eta^{B 1} V_{t} d t+\sqrt{V_{t}} d B_{t}^{(1)}\right) \\
& +\sigma_{V} \sqrt{1-\rho^{2}} g_{v}^{(i)}\left(\eta^{B 2} V_{t} d t+\sqrt{V_{t}} d B_{t}^{(2)}\right) \\
& +\left(\sum_{j, k} \Delta^{(j, k)} g^{(i)} d N_{t}^{(j, k)}-E^{P}\left[\Delta g^{(i)}\right] \lambda^{P} V_{t} d t\right) \\
& +\left(E^{P}\left[\Delta g^{(i)}\right] \lambda^{P}-E^{Q}\left[\Delta g^{(i)}\right] \lambda^{Q}\right) V_{t} d t
\end{aligned}
$$

where the exposures to stock price diffusion risk, volatility diffusion risk, and jump risk are given by

$$
\begin{aligned}
g_{s}^{(i)} & =\left.\frac{\partial g^{(i)}(s, v)}{\partial s}\right|_{\left(S_{t}, V_{t}\right)} \\
g_{v}^{(i)} & =\left.\frac{\partial g^{(i)}(s, v)}{\partial v}\right|_{\left(S_{t}, V_{t}\right)} \\
\Delta^{(j, k)} g^{(i)} & =g^{(i)}\left(\left(1+x^{(j)}\right) S_{t^{-}}, V_{t^{-}}+y^{(k)}\right)-g^{(i)}\left(S_{t^{-}}, V_{t^{-}}\right) .
\end{aligned}
$$

For standard European call and put options these expressions can be calculated using the option pricing model in Duffie, Pan, and Singleton (2000). In contrast to Liu and Pan 
(2003), closed form solutions for the Fourier transforms needed to price options are no longer available. However, the numerical evaluation of the respective differential equations is rather straightforward.

\section{Portfolio Planning Problem}

The objective of the investor is to maximize the expected utility of terminal wealth, i.e. there is no intermediate consumption. The assumed utility function is of the CRRA type with risk aversion parameter $\gamma$. The tradable assets in our economy are the money market account, which earns interest at the constant rate $r$, the stock, and a sufficient number of derivative assets written on the stock, so that the market is complete. Kraft (2003) and Korn and Kraft (2004) focus on the technical aspects of such continuous-time asset allocation problems in the context of stochastic investment opportunity sets.

The investor's optimization problem and its solution are structurally similar to Liu and Pan (2003). Compared to their paper we present a significant generalization of the jump component in the dynamics of the state variables. First, we include jumps in volatility in addition to price jumps, and, second, we allow for non-deterministic jump sizes. As a result, our model represents an economy where jumps may occur in the stock price only, in the variance only, or in both processes simultaneously. The structure of the jump size distribution shown in Table 1 makes it possible to generate different correlation structures for the jump sizes.

Let $\phi_{t}$ and $\psi_{t}^{(i)}, i=1,2 \ldots, M$, represent the fractions of wealth invested in the stock and in the $M$ derivative assets, respectively. In the case that $\phi_{t}+\sum_{i=1}^{M} \psi_{t}^{(i)} \neq 1$ the remaining wealth is invested in the money market account. The stochastic differential equation for wealth is then given by

$$
d W_{t}=W_{t}\left\{\left(1-\phi_{t}-\sum_{i=1}^{M} \psi_{t}^{(i)}\right) r d t+\phi_{t} \frac{d S_{t}}{S_{t}}+\sum_{i=1}^{M} \psi_{t}^{(i)} \frac{d O_{t}^{(i)}}{O_{t}^{(i)}}\right\} .
$$

For the following analysis, it is useful to work with exposures to the fundamental risk factors $B^{(1)}, B^{(2)}$, and to the $M-1$ different jump events instead of portfolio weights. Rewriting the dynamics of wealth in terms of these exposures, one obtains

$$
\begin{aligned}
d W_{t}= & r W_{t} d t+\theta_{t}^{B 1} W_{t}\left(\eta^{B 1} V_{t} d t+\sqrt{V_{t}} d B_{t}^{(1)}\right)+\theta_{t}^{B 2} W_{t}\left(\eta^{B 2} V_{t} d t+\sqrt{V_{t}} d B_{t}^{(2)}\right) \\
& +W_{t}\left(\sum_{j, k} \theta_{t}^{N^{(j, k)}} d N_{t}^{(j, k)}-\sum_{j, k} q_{j k} \theta_{t}^{N^{(j, k)}} \lambda^{Q} V_{t} d t\right) .
\end{aligned}
$$

The fraction of wealth $\theta_{t}^{B 1}$ invested in risk factor $\sqrt{V_{t}} d B_{t}^{(1)}$ is related to the investment in the traded assets via

$$
\theta_{t}^{B 1}=\phi_{t}+\sum_{i=1}^{M} \psi_{t}^{(i)}\left(\frac{g_{s}^{(i)} S_{t}}{O_{t}^{(i)}}+\sigma_{V} \rho \frac{g_{v}^{(i)}}{O_{t}^{(i)}}\right)
$$


Analogously, we find

$$
\theta_{t}^{B 2}=\sigma_{V} \sqrt{1-\rho^{2}} \sum_{i=1}^{M} \psi_{t}^{(i)} \frac{g_{v}^{(i)}}{O_{t}^{(i)}}
$$

with $\theta_{t}^{B 2}$ representing the fraction of wealth invested in $\sqrt{V_{t}} d B_{t}^{(2)}$, and

$$
\theta_{t}^{N^{(j, k)}}=\phi_{t} x^{(j)}+\sum_{i=1}^{M} \psi_{t}^{(i)} \frac{\Delta^{(j, k)} g^{(i)}}{O_{t}^{(i)}}
$$

where $\theta_{t}^{N^{(j, k)}}$ stands for the fraction of wealth invested in the risk factor $d N_{t}^{(j, k)}$ with jump sizes $\left(x^{(j)}, y^{(k)}\right)$. So $\theta_{t}^{N^{(j, k)}}$ gives the relative jump in wealth if there is a jump of size $x^{(j)}$ in the stock price and of size $y^{(k)}$ in volatility. For example, $\theta_{t}^{N^{(j, k)}}<0$ means that the investor's wealth will decrease by $\theta_{t}^{N^{(j, k)}} \cdot 100$ percent when a jump of type $(j, k)$ occurs. This notation differs from the one used in Liu and Pan (2003) in that the sign of $\theta_{t}^{N}$ immediately indicates the direction of the wealth change in case of a jump.

When the market is complete, any exposure $\left(\theta_{t}^{B 1}, \theta_{t}^{B 2}, \theta_{t}^{N^{(j, k)}}\right)$ can be obtained by suitable positions in the stock, the money market account, and the contingent claims. The positions in the traded assets follow from solving the system of equations $(6),(7)$ and (8) for $\phi_{t}$ and $\psi_{t}^{(i)}(i=1,2, \ldots, M)$.

The investor's optimization problem is given by

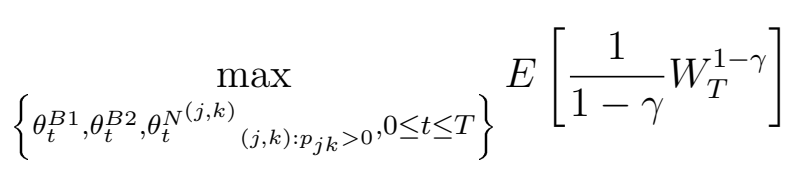

subject to the wealth dynamics in (5). The associated indirect utility function $J(t, w, v)$ is then obtained as

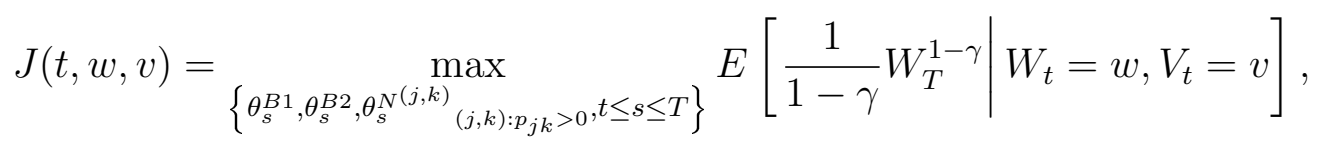

again subject to (5). From this we can immediately derive the Hamilton-Jacobi-Bellman (HJB) equation:

$$
\begin{aligned}
& \max _{\left\{\theta_{t}^{B 1}, \theta_{t}^{B 2}, \theta_{t}^{N(j, k)}\right.}\left\{J_{t}+w J_{W}\left(r+\theta_{t}^{B 1} \eta^{B 1} v+\theta_{t}^{B 2} \eta^{B 2} v-\sum_{j, k} q_{j k} \theta_{t}^{N^{(j, k)}} \lambda^{Q} v\right)\right. \\
& +\frac{1}{2} w^{2} J_{W W} v\left[\left(\theta_{t}^{B 1}\right)^{2}+\left(\theta_{t}^{B 2}\right)^{2}\right] \\
& +\lambda^{P} v \sum_{j, k} p_{j k}\left[J\left(t, w\left(1+\theta_{t}^{N^{(j, k)}}\right), v+y^{(k)}\right)-J(t, w, v)\right] \\
& +\left[\kappa^{P}\left(\bar{v}^{P}-v\right)-E^{P}[Y] \lambda^{P} v\right] J_{V}+\frac{1}{2} \sigma_{V}^{2} v J_{V V} \\
& \left.+\sigma_{V} v w J_{W V}\left(\rho \theta_{t}^{B 1}+\sqrt{1-\rho^{2}} \theta_{t}^{B 2}\right)\right\}=0
\end{aligned}
$$


where subscripts of $J$ denote partial derivatives.

To find the optimal portfolio composition, one first needs to know the indirect utility function $J$. The usual guess for this function, as in Liu and Pan (2003) and numerous other papers, is

$$
J(t, w, v)=\frac{w^{1-\gamma}}{1-\gamma} \exp \{\gamma h(\tau)+\gamma H(\tau) v\},
$$

where $\tau=T-t$. After computing the necessary partial derivatives one can deduce the optimal exposures $\theta_{t}^{* B 1}, \theta_{t}^{* B 2}$, and $\theta_{t}^{* N^{(j, k)}}$ to the fundamental risk factors $\sqrt{V_{t}} d B_{t}^{(1)}$, $\sqrt{V_{t}} d B_{t}^{(2)}$, and jumps of size $x^{(j)}$ in the stock price and $y^{(k)}$ in volatility from the standard first-order conditions. They are given in the following lemma.

Lemma 1 (Optimal exposures to fundamental risk factors) The optimal exposures to the fundamental risk factors are given by

$$
\begin{aligned}
\theta_{t}^{* B 1}= & \frac{\eta^{B 1}}{\gamma}+\rho \sigma_{V} H(\tau) \\
\theta_{t}^{* B 2}= & \frac{\eta^{B 2}}{\gamma}+\sqrt{1-\rho^{2}} \sigma_{V} H(\tau) \\
\theta_{t}^{* N^{(j, k)}}= & {\left[\left(\frac{p_{j k} \lambda^{P}}{q_{j k} \lambda^{Q}}\right)^{1 / \gamma}-1\right]+\left(\frac{p_{j k} \lambda^{P}}{q_{j k} \lambda^{Q}}\right)^{1 / \gamma}\left[e^{H(\tau) y^{(k)}}-1\right] } \\
& \text { with } \theta_{t}^{* N^{(j, k)}} \geq-1 \text { for all } j, k .
\end{aligned}
$$

Note the additional restriction for $\theta_{t}^{* N^{(j, k)}}$, which assures that wealth cannot become negative. The optimal portfolio weights for the risky assets follow immediately from these optimal weights via Equations (6), (7) and (8).

Finally, the Expressions (11), (12), and (13) are plugged back into the HJB equation (9). Collecting terms with and without $v$ one obtains the following system of ordinary differential equations for the functions $h$ and $H$ :

$$
\begin{aligned}
h^{\prime}(\tau) & =\kappa^{P} \bar{v}^{P} H(\tau)+\frac{1-\gamma}{\gamma} r \\
H^{\prime}(\tau) & =a+b H(\tau)+c H^{2}(\tau)+\lambda^{Q} \sum_{j, k} q_{j k}\left[\left(\frac{p_{j k} \lambda^{P}}{q_{j k} \lambda^{Q}}\right)^{1 / \gamma} \exp \left\{y^{(k)} H(\tau)\right\}\right]
\end{aligned}
$$

with the boundary conditions $h(0)=H(0)=0$ and

$$
\begin{aligned}
a & =\frac{1-\gamma}{2 \gamma^{2}}\left[\left(\eta^{B 1}\right)^{2}+\left(\eta^{B 2}\right)^{2}\right]+\frac{1-\gamma}{\gamma} \lambda^{Q}-\frac{1}{\gamma} \lambda^{P} \\
b & =-\left(\kappa^{P}+E^{P}[Y] \lambda^{P}\right)+\frac{1-\gamma}{\gamma} \sigma_{V}\left(\rho \eta^{B 1}+\sqrt{1-\rho^{2}} \eta^{B 2}\right) \\
c & =\frac{1}{2} \sigma_{V}^{2} .
\end{aligned}
$$


In Liu and Pan (2003), $Y$ is identically equal to zero, and the partial differential equation (15) for $H$ is a Ricatti equation with constant coefficients. In this case, a closed form solution is available for both $H$ and $h$. This is no longer true in the general case where the volatility jump size is different from zero or even stochastic. Now, the system $(14,15)$ has to be solved numerically, e.g. via the Runge-Kutta method.

Nevertheless, our function $H$ shares an important property with the corresponding function in Liu and Pan (2003), namely that $H(\tau) \geq 0$ for $\gamma<1, H(\tau) \leq 0$ for $\gamma>1$, and $H(\tau)=0$ for the $\log$-investor with $\gamma=1$. A proof is given in Appendix A.

This property of $H$ implies that the indirect utility of the investor is increasing in $V$. To get the intuition, note that we have made the by now standard assumption that the size of the market price of risk is increasing in $V$ for all risk factors. The higher $V$, the larger the compensation earned by the investor per unit of risk, and the larger therefore his utility.

In the economy analyzed by Liu, Longstaff, and Pan (2003) only the stock and the money market account are traded, which is in our case equivalent to imposing the additional restrictions $\theta_{t}^{* B 1}=\phi_{t}, \theta_{t}^{* N^{(j, k)}}=\phi_{t} x^{(j)}$ and $\theta_{t}^{* B 2}=0$ when solving for the optimal exposure. Without derivatives, the exposure to the second diffusion risk with impact on volatility only is equal to zero, and the relation between the exposure to the first diffusion risk and to jump risk is fixed at the relation of these risk factors for the stock. The introduction of derivatives completes the market and allows the investor to trade the risk factors separately. In Section 5, we will analyze the economic value of this possibility.

The optimal demand in (11), (12), and (13) has two basic sources which were already discussed by Merton (1971). First, the investor wants to earn the risk premium for the respective factor, which represents the myopic or speculative demand, given by the first summand. Second, there is also a desire to hedge against unfavorable changes in the state variables determining the investment opportunity set, i.e. in our framework against changes in $V$. This is the investor's hedging demand, given by the second summand.

The most important difference between a model that allows for jumps in volatility and the setup studied by Liu and Pan (2003) where jumps affect the stock price only is that the optimal demand for jump risk now contains a hedging component which is not present in an economy without variance jumps. This hedging demand is given by the second summand in Equation (13). For $y^{(k)}>0$, there will thus be demand for jump risk $\left(\theta_{t}^{* N^{(j, k)}} \neq 0\right)$ even in the case when $\lambda^{P} p_{j k}=\lambda^{Q} q_{j k}$, i.e. when the jump risk premium is zero. To get the intuition, note that the hedging demand arises because the investor wants to hedge against unfavorable changes in volatility. Expressing this in terms of exposures to risk factors, there will thus be a hedging demand in all factors that have an impact on volatility. For a jump with $y^{(k)} \neq 0$, this generates a hedging demand in this jump risk factor.

The hedging demand of the investor arises from the impact of $V$ on the compensation per unit of risk, as, e.g. discussed in Munk (2004) or Munk and Sørensen (2004). For small $V$, the expected return earned by the investor on his myopic exposure is low, and the risk of a large negative return and thus a low terminal wealth is comparably high. This induces 
the investor to shift wealth from states with high $V$ to states with low $V$. On the other hand, for high $V$, the expected return is high. This induces the investor to shift wealth to states with a high $V$ to grasp these good investment opportunities. Taken together, his ultimate hedging demand depends on the trade-off between these two opposite effects. As described in Liu (2001), for $\gamma>1$, the utility function of an investor is unbounded from below, but bounded from above. He cares more about states with low $V$ since this implies a higher probability of losses due to a lower expected return. Thus, his hedge is to take a short position in $V$. In line with this intuition, the marginal indirect utility $J_{W}$ of the investor is decreasing in $V$, which formally follows from the fact that $H$ is non-positive. A low volatility thus corresponds to a high indirect utility, implying a 'bad state'. For $\gamma<1$, on the other hand, the utility function of the investor is bounded from below and unbounded from above, and he speculates by taking a long position in volatility. Finally, for $\gamma=1$, both effects exactly offset each other, and the investor is neutral with respect to changes in the investment opportunity set.

In contrast to Liu and Pan (2003) this hedging demand can now be met by a position in all risk factors and not only in the two diffusive risks. Consider the case $\gamma>1$. $H$ is non-positive and the investor wants to hedge by taking a short position in variance. The hedging demand for the first diffusion $d B^{(1)}$ will be positive if $\rho<0$, and the hedging demand for the second diffusion $d B^{(2)}$ will be negative. Given that any jump in volatility is an upward jump, the hedging demand for all jumps that also affect volatility will be negative, while there will be no hedging demand for jumps that occur only in the stock price. The analysis for $\gamma<1$ proceeds along the same lines, showing that the investor now hedges by taking a long position in variance, which results in a positive hedging demand for jump risk.

To explain the structure of the jump demand in our model in more detail, consider two pairs of jump size realizations $\left(x^{(a)}, y^{(a)}\right)$ and $\left(x^{(b)}, y^{(a)}\right)$ with $p_{a a}=p_{b a}=0.5$. To focus on price jumps the volatility jump size is the same for both pairs. Assume $\gamma>1$, $x^{(b)}<x^{(a)}<0, y^{(a)}>0$ and $\lambda^{P}=\lambda^{Q}$, i.e. there is no jump intensity premium.

If $p_{a a} / q_{a a}=p_{b a} / q_{b a}=1$, the jump size premium is also zero. There is no myopic demand here, and the negative hedging demand is equal for both jump realizations, i.e. $\theta_{t}^{N^{(a, a)}}=\theta_{t}^{N^{(b, a)}}<0$. For the hedging demand, it is thus only the size of $y^{(1)}$ that matters. Note that there is no insurance against price jumps, but the investor is willing to give up wealth in situations when volatility has jumped upwards.

Now let $p_{a a} / q_{a a}>1>p_{b a} / q_{b a}$. This means that the jump size premium is relatively higher for large negative jumps, so that insurance against these jumps is relatively more expensive. This corresponds to the stylized facts reported in the literature, namely that out-of-the-money put options seem very expensive. In this scenario we obtain a positive myopic demand for jump type $(a, a)$ and a negative myopic demand for jump type $(b, a)$, where the investor earns a risk premium in both cases. The hedging demand is still negative for both jump realizations. In absolute terms it is larger than in Case I for jump $(a, a)$ and smaller for jump $(b, a)$. In total there is definitely no insurance against large jumps, since $\theta_{t}^{N^{(b, a)}}<0$, but the sign of $\theta_{t}^{N^{(a, a)}}$ is not determined. 
When $p_{a a} / q_{a a}<1<p_{b a} / q_{b a}$, the situation is exactly reversed. The total position of the investor will not contain protection against small jumps, but there may be insurance against large negative jumps, depending on the trade-off between risk premium and additional utility derived from hedging.

\section{Numerical Example: Deterministic Jumps}

For the numerical example, we focus on the framework used for the examples in Liu, Longstaff, and Pan (2003), where the jump sizes for both the asset price and the variance are deterministic, i.e. we set $X \equiv \mu_{X}$ and $Y \equiv \mu_{Y}$. This also implies that the jump size distribution degenerates into a single point, which implies that the pricing of jump risk depends on the difference between $\lambda^{P}$ and $\lambda^{Q}$ only, while the jump size distributions are degenerate. Assuming the usual case of a negative jump size for prices $\left(\mu_{X}<0\right)$ and upward jumps in volatility $\left(\mu_{Y}>0\right)$, a jump decreases prices and simultaneously increases volatility, which can be regarded as an increase in uncertainty after a market crash.

Due to the simpler structure of the model compared to the general case, now only two non-redundant derivatives are needed to complete the market. Liu and Pan (2003) show that the market is complete if the claims satisfy the restriction $D_{t} \neq 0$, where

$$
D_{t}=\left(\frac{\Delta g^{(1)}}{\mu_{X} O_{t}^{(1)}}-\frac{g_{s}^{(1)} S_{t}}{O_{t}^{(1)}}\right) \frac{g_{v}^{(2)}}{O_{t}^{(2)}}-\left(\frac{\Delta g^{(2)}}{\mu_{X} O_{t}^{(2)}}-\frac{g_{s}^{(2)} S_{t}}{O_{t}^{(2)}}\right) \frac{g_{v}^{(1)}}{O_{t}^{(1)}}
$$

and $\Delta g^{(i)}=g^{(i)}\left(\left(1+\mu_{X}\right) S_{t^{-}}, V_{t^{-}}+\mu_{Y}\right)-g^{(i)}\left(S_{t^{-}}, V_{t^{-}}\right)$. This condition says that the determinant of the local sensitivity matrix of the two derivative contracts must not be zero, implying that the two contracts are linearly independent.

The transformation from risk exposures to asset positions works as in Liu and Pan (2003). For the risk exposure to the deterministic jumps, we now take the simpler notation $\theta_{t}^{N}$ instead of $\theta_{t}^{N^{(j, k)}}$. Solving Equations (6), (7) and (8) for the optimal positions in the stock and in the derivative assets gives:

$$
\begin{aligned}
\phi_{t} & =\theta_{t}^{B 1}-\sum_{i=1}^{2} \psi_{t}^{(i)}\left(\frac{g_{s}^{(i)} S_{t}}{O_{t}^{(i)}}+\sigma_{V} \rho \frac{g_{v}^{(i)}}{O_{t}^{(i)}}\right) \\
\psi_{t}^{(1)} & =\frac{1}{D_{t}}\left[\frac{g_{v}^{(2)}}{O_{t}^{(2)}}\left(\frac{\theta_{t}^{N}}{\mu_{X}}-\theta_{t}^{B 1}+\frac{\theta_{t}^{B 2} \rho}{\sqrt{1-\rho^{2}}}\right)-\frac{\theta_{t}^{B 2}}{\sigma_{V} \sqrt{1-\rho^{2}}}\left(\frac{\Delta g^{(2)}}{\mu_{X} O_{t}^{(2)}}-\frac{g_{s}^{(2)} S_{t}}{O_{t}^{(2)}}\right)\right] \\
\psi_{t}^{(2)} & =\frac{1}{D_{t}}\left[\frac{\theta_{t}^{B 2}}{\sigma_{V} \sqrt{1-\rho^{2}}}\left(\frac{\Delta g^{(1)}}{\mu_{X} O_{t}^{(1)}}-\frac{g_{s}^{(1)} S_{t}}{O_{t}^{(1)}}\right)-\frac{g_{v}^{(1)}}{O_{t}^{(1)}}\left(\frac{\theta_{t}^{N}}{\mu_{X}}-\theta_{t}^{B 1}+\frac{\theta_{t}^{B 2} \rho}{\sqrt{1-\rho^{2}}}\right)\right]
\end{aligned}
$$

Note the typo in Liu and Pan (2003), where the term $\theta_{t}^{B 2} \rho / \sqrt{1-\rho^{2}}$ is subtracted rather than added in both the second and the third equation.

One of the main items of interest in our paper is the impact of variance jumps on the structure of the optimal demand functions. To analyze this impact, we first perform 
a sensitivity analysis with respect to the deterministic volatility jump size $\mu_{Y}$. All other parameters are unchanged, which implies in particular that the variance of variance and the mean variance will change. Therefore, we refer to this analysis as the uncalibrated case. The key new result derived in Section 3 is that with jumps in volatility, the jump demand exhibits a hedging component in addition to the familiar speculative part. Since the size and direction of hedging demand primarily depends on the function $H(\tau)$, we first take a closer look at the impact of the variance jump size on this function.

The comparison is based on the benchmark parametrization in Liu, Longstaff, and Pan (2003). In our notation, the parameters are $\kappa^{P}=5.3, \bar{v}^{P}=\frac{0.11512}{5.3}=0.021721$, $\sigma_{V}=0.22478, \rho=-0.57, \lambda^{P}=1.84156, \mu_{X}=-0.25$, and $\mu_{Y}=0.22578$. Furthermore, we assume that jump risk and diffusion risk each account for half of the expected excess return on the stock, and we choose $\eta^{B 2}=-2.0$, where the negative sign can be justified based on the discussion in Liu and Pan (2003). This yields the following values for the other parameters in the model: $\kappa^{Q}=2.40292, \bar{v}^{Q}=0.04791, \lambda^{Q}=11.64964$ and $\eta^{B 1}=2.45112$. Table 2 summarizes these values as Parametrization I.

Figure 1 shows the function $H$ as a function of the planning horizon $\tau$ for the benchmark case $\mu_{Y}=0.22578$ as well as for $\mu_{Y}=0.0$ and $\mu_{Y}=0.1$. The coefficient of risk aversion is equal to 3. For all three values of $\mu_{Y}$, the function $H$ is zero for $\tau=0$ and decreases sharply for short planning horizons. It then approaches an 'asymptotic' value for planning horizons of more than one year, where the asymptotic value is determined by the parameters. The hedging demand, which depends on the planning horizon only through $H$, is thus zero for $\tau=0$ and stabilizes at some value for increasing time to maturity. Intuitively, this can be explained by the existence of some 'upper bound' on the hedging demand of the investor. Even if the investor is risk averse, he is still willing to take some risk to earn the risk premia.

The absolute value of $H$ is increasing in $\mu_{Y}$, so that the hedging demand is also increasing in $\mu_{Y}$. Intuitively, an increase in the variance jump size increases the variance of variance. The higher variance risk then leads to a higher hedging demand against this risk factor. This is not only true for the hedging demand in jump risk, but also for the hedging demand in diffusion risk, which can be explained by a higher overall concern of the investor about variance risk. Note that the analysis performed here is a sensitivity analysis in which we assume that only $\mu_{Y}$ changes.

In the next step, we investigate the economic consequences of the presence of a jump component in the volatility process, i.e. the impact of variance jumps and their size on optimal exposures to risk factors and optimal portfolio decisions. Again, we consider the case of jumps with deterministic sizes $\mu_{X}$ and $\mu_{Y}$. As in the above analysis $\mu_{Y}$ will be taken from the set $\{0,0.1,0.22578\}$. However, now we will not simply leave all other parameters unchanged when we compare economies with volatility jumps to one without. When varying $\mu_{Y}$ one has to make sure that the market information the investor could use to calibrate the model of his choice is correctly represented in the parametrization. This is what we call the calibrated case. For example, the parameters plugged in the model chosen by the investor must result in correct values for expected stock returns, option prices, or risk premia. In more detail, the following parameters were restricted to 
be identical across models: the instantaneous expected excess return on the stock (given by $\left(\eta^{B 1}+\mu_{X}\left(\lambda^{P}-\lambda^{Q}\right)\right) \bar{v}^{P}$ in all of the models), the instantaneous variance of stock returns (given by $\bar{v}^{P}+\mu_{X}^{2} \lambda^{P} \bar{v}^{P}$ in the two models with volatility jumps and by $\bar{v}^{P}$ in the one without), the instantaneous variance of variance (given by $\sigma_{V}^{2} \bar{v}^{P}+\mu_{Y}^{2} \lambda^{P} \bar{v}^{P}$ in the models with volatility jumps and by $\sigma_{V}^{2} \bar{v}^{P}$ in the one without), the average time between two jumps (given by $\left(\lambda^{P} \bar{v}^{P}\right)^{-1}$ in all models), and the relative jump size in the stock price (given by $\mu_{X}$ in all models). Furthermore, we assume that $V_{0}$ is the same in all models and equal to $\bar{v}^{P}$. To ultimately calibrate the model, three additional option prices are needed. We use two European call options with a time to maturity of three months and strike prices equal to $90 \%$ and $100 \%$ of the initial stock price. The third option is a European call with one month to maturity and a strike price equal to $90 \%$ of the initial stock price. The prices of these options are computed based on the model developed by Duffie, Pan, and Singleton (2000).

As above, the benchmark case is given by the model and the associated parameters described in Liu, Longstaff, and Pan (2003). Table 2 shows the benchmark case, denoted by Parametrization I, as well as Parametrization II ( $\left.\mu_{Y}=0.1\right)$, and Parametrization III $\left(\mu_{Y}=0\right)$. The scenario $\mu_{Y}=0.1$ was included to represent the case, where the investor correctly assumes that volatility can jump, but uses a wrong jump size. Due to the fact that jumps are rare, the moments of the jump size are subject to severe estimation risk, so that such an error can occur easily. In general, with a decreasing volatility jump size $\mu_{Y}$, the calibration yields a lower speed of mean reversion $\kappa^{P}$ and a higher level of the volatility of volatility $\sigma_{V}$. This result can be explained intuitively by noting that, with smaller jumps in volatility, the instantaneous variance of variance has to be generated to a larger degree (or even completely) by the diffusive part of the volatility dynamics. This results in higher levels of $\sigma_{V}$ and lower levels of $\kappa^{P}$.

We assume that the derivatives used by the investor to form his portfolio are the two 3 -month call options described above. Our choice of these options is motivated by two considerations. First, options with these characteristics are usually highly liquid. Second, an ATM call is a standard choice for a volatility sensitive instrument, while the option with the lower strike price has a large exposure to jump risk.

We first analyze the properties of the function $H(\tau)$ for different values of $\mu_{Y}$. From Figure 2 we can see that the impact of $\tau$ is quite similar to the case analyzed in Figure 1. Again, $H(\tau)$ is zero for $\tau=0$ and increases sharply in absolute terms for increasing $\tau$, before it approaches an asymptotic value. The approximation to this asymptotic value is much slower for $\mu_{Y}=0.0$ than for the benchmark case of $\mu_{Y}=0.22578$. This can be explained by the smaller speed of mean reversion of volatility, due to which it takes longer for volatility shocks to die out.

The impact of $\mu_{Y}$, however, is significantly different depending on whether we only vary $\mu_{Y}$ as in Figure 1 or recalibrate the model as in Figure 2. While in the first case, $H(\tau)$ in increasing in absolute value in $\mu_{Y}$, it is now decreasing in $\mu_{Y}$. To get the intuition, note that we fix the variance of variance at some level. If we wrongly assume a model without jumps in volatility, then the whole variance of variance has to be explained by the diffusion components, and the hedging demand of the investor in the diffusion component 
increases, which implies that $H(\tau)$ increases in absolute value for $\mu_{Y}$ decreasing.

In a second step, we analyze the impact of $\mu_{Y}$ on the decisions made by the investor. The optimal exposures are given in (11), (12) and (13). For the special case $Y=\mu_{Y}=0.0$ and $X=\mu_{X}$, the expressions coincide with the formulas given in Liu and Pan (2003), where the function $H(\tau)$ can be obtained in closed form. The conversion of optimal exposures into optimal asset positions $\psi^{(1)}, \psi^{(2)}$, and $\phi_{t}$ works as described above via Equations (17), (18) and (19). Note, however, that the optimal exposures represent the basic result, whereas the optimal asset positions are the 'derived' result. These numbers naturally depend on the choice of assets, and there is no unique representation. So our results concerning the stock and the two calls represent only one possible example for the portfolio composition. Nevertheless, in reality the investor has to buy (or sell) assets to achieve the desired exposure, so that differences with respect to this output of the model actually describe the differences in behavior one would see in the real world.

Figure 3 shows the optimal exposures to the fundamental risk factors for varying time horizons and different values of $\mu_{Y}$. For very short horizons the optimal exposures almost exclusively reflect myopic demand, so all differences between the parametrizations can be attributed to the different risk premium for $\sqrt{V} d B^{(2)}$. Note that the risk premia for the other two risk factors coincide for all calibrations by assumption. When $\mu_{Y}=0.0$, all the variance risk is attributed to the two diffusion risk factors, which increases the hedging component of the demand compared to the cases with $\mu_{Y}>0$. On the other hand, there is only myopic demand in jump risk, so that the planning horizon is irrelevant for the optimal exposure. The larger $\mu_{Y}$, the more important jump risk is for hedging volatility risk, and the less important are the diffusions. Consequently, the optimal exposure to jump risk depends more on the planning horizon, while the dependence of the optimal exposure to the two diffusions on the planning horizon becomes less pronounced. Finally, with increasing planning horizon the optimal exposures are more or less constant for all three values of $\mu_{Y}$. The investor tends to change his portfolio allocations to a smaller degree when the investment horizon is still long.

The picture changes when we look at asset positions which are given in Figure 4. Now, the differences between the three models are much more pronounced. The positions in the assets vary significantly for $\tau=0$, despite the fact that the optimal exposures to the risk factors $d B^{(1)}$ and to jump risk are the same. This can be attributed to the fact that the sensitivities of the derivatives are calculated in different models and vary substantially. The value of $\mu_{Y}$ has a significant impact on the optimal portfolio. For $\mu_{Y}=0.0$, e.g., the optimal position in the stock is negative for longer planning horizons, while it is positive in the other two cases with $\mu_{Y}>0$. Finally, for the optimal holdings of the traded assets, we observe that for longer planning horizons the optimal positions tend towards an 'asymptotic' value which again depends on $\mu_{Y}$. 


\section{Economic Value of Derivatives}

In this section we assess the economic value of derivatives. As stated in the introduction, we augment the choice set of the investor relative to the analysis in Liu, Longstaff, and Pan (2003), since now all the necessary market completing derivatives are assumed to be traded. A comparison to the case where only the stock and the money market account are traded allows us to assess the economic value of trading derivatives, thus extending the analysis of Liu and Pan (2003) to the case of jumps in volatility. Clearly, this represents a first step, since we perform the analysis in a partial equilibrium context, where the prices of assets already traded are implicitly assumed to remain unchanged. As discussed above derivatives allow the investor to trade the risk factors separately. Compared to a situation where he can only trade the stock and the money market account, his utility will thus necessarily increase.

To measure the economic value of derivatives, we use the portfolio improvement measure $\mathcal{R}^{\mathcal{W}}$ as proposed by, among others, Liu and Pan (2003). It is defined as the annualized percentage difference in certainty equivalent wealth

$$
\mathcal{R}^{\mathcal{W}}=\frac{\ln (\mathcal{W} / \widehat{\mathcal{W}})}{T}
$$

where $\mathcal{W}(\widehat{\mathcal{W}})$ is the certainty equivalent wealth for the case with (without) derivatives. $\mathcal{W}$ and $\widehat{\mathcal{W}}$ are defined implicitly via

$$
J\left(0, W_{0}, V_{0}\right)=\frac{\mathcal{W}^{1-\gamma}}{1-\gamma}
$$

and

$$
\widehat{J}\left(0, W_{0}, V_{0}\right)=\frac{\widehat{\mathcal{W}}^{1-\gamma}}{1-\gamma}
$$

with $J(\widehat{J})$ representing the indirect utility function with (without) derivatives. Since the investor has constant relative risk aversion, $\mathcal{R}^{\mathcal{W}}$ does not depend on his initial wealth $W_{0}$.

As in Section 4 we analyze the special case with simultaneous and deterministic jumps in the stock and in volatility. The indirect utility function $\widehat{J}$ can be computed as in Liu, Longstaff, and Pan (2003), while for the computation of $J$ we first need to solve Equations (14) and (15) numerically and then plug the solution to this system into (10). The comparison between the situations with and without derivatives is based on Parametrization I from Table 2. Derivatives allow the investor to achieve his optimal exposure to the individual risk factors. Without derivatives, his exposure to the second diffusion factor $B^{(2)}$ is zero, and the relative exposure to diffusion risk $B^{(1)}$ and jump risk is fixed at the relation of these two risk factors in the stock.

Figure 5 illustrates the portfolio improvement $\mathcal{R}^{\mathcal{W}}$ as a function of the planning horizon, the speed of mean reversion, the jump risk premium (captured by the relation of the risk-neutral and the true jump intensity), and the variance jump size. The upper left 
graph shows the portfolio improvement measure $\mathcal{R}^{\mathcal{W}}$ for varying planning horizon $\tau$. For $\tau=0$, there is only myopic demand, and the portfolio improvement of $3 \%$ arises from the ability of the investor to achieve this optimal demand. For increasing time horizons, there is an additional gain from achieving the optimal hedging demand, too, and the portfolio improvement stabilizes at nearly $5.5 \%$ for a horizon of more than 2 years.

The upper right graph shows the impact of the speed of mean reversion on the portfolio improvement. The higher $\kappa^{P}$, the less impact shocks in variance have, and the lower the variance of variance. Consequently, hedging becomes less important for increasing $\kappa^{P}$. For $\kappa^{P}$ between one and two, the optimal demand of the investor which can be reached by trading derivatives is the most different from the risk package offered by the stock and the money market account only. For other values of $\kappa^{P}$, the change in the absolute hedging demand lowers this difference between optimal exposure and exposure attainable without trading derivatives, so that the portfolio improvement becomes smaller. For very high values of $\kappa^{P}$, the hedging demand goes to zero, and the portfolio improvement can be attributed to the possibility to achieve the optimal myopic exposure, only.

The impact of the risk-neutral jump intensity $\lambda^{Q}$ is shown in the lower left graph. The more $\lambda^{Q}$ differs from the (fixed) $\lambda^{P}$, the larger the compensation for a position in jump risk. The impact of a given difference between the optimal exposure and the package offered by the stock on the portfolio improvement thus increases. Furthermore, a change in $\lambda^{Q}$ changes the optimal myopic exposure to jump risk and the hedging exposure to all three risk factors. Similar to the discussion for $\kappa^{P}$, the portfolio improvement tends to increase in the differences between the resulting optimal exposure and the package offered by the stock. In our example, the minimal improvement is realized for $\lambda^{Q} / \lambda^{P} \approx 1.6$.

Finally, the lower right graph shows the portfolio improvement for a varying variance jump size $\mu_{Y}$. The larger $\mu_{Y}$, the larger the variance of variance, and the larger the investor's hedging demand. Trading in derivatives, which allows him to meet this hedging demand in particular in jump risk, thus becomes more valuable, and the portfolio improvement increases in $\mu_{Y}$.

\section{Model Mis-Specification}

We now investigate the consequences of model mis-specification in the context of volatility jumps. Note that this mis-specification can go two ways. The investor either uses a model that is 'too small', e.g. by ignoring volatility jumps, or one that is 'too large', e.g. by including such jumps although they are not a part of the true model. We have seen in Section 4 that asset positions and optimal exposures to risk factors usually change noticeably when different models are used. However, the ultimate measure for the impact of model mis-specification is the loss in utility the investor has to suffer when using incorrect dynamics for the stock price or for volatility. Again the analysis considers the case of deterministic jumps, and the different models are calibrated to the same set of prices, risk premia, and moments for the stock price and its variance. 
To analyze the impact of model mis-specification, we proceed in several steps. First, we calculate the (seemingly) optimal exposure to the risk factors, derived from the improper model. Then these exposures are transformed into asset demands, using Equations (17), (18) and (19), with the sensitivities still based on the incorrect model. This gives the portfolio the investor will actually buy at the market. In the final step, the asset positions are converted back into realized exposures, now based on the sensitivities in the true model, using Equations (6), (7) and (8). Note that this last step in the calculation can only be done to analyze the impact of model mis-specification, but not by the investor who does not know the true model.

First, we consider the situation where the true model is given by Equations (1) and (2) and includes volatility jumps, but where the investor bases his decision on a model without jumps in volatility. The true model is thus given by Parametrization I from Table 2, while the investor ignores volatility jumps, i.e. uses Parametrization III from Table 2. Figure 6 shows the realized exposures as a function of the planning horizon, where we have set the local variance equal to its long run mean $\bar{v}^{P}$. A comparison of this figure with the (seemingly) optimal exposures in the lower graph of Figure 3 and the truly optimal exposure in the upper graph shows that the use of the wrong sensitivities can have a significant impact. In particular, in the correct model with $\mu_{Y}=0.22578$, the optimal exposure to jump risk is increasing in the planning horizon (in absolute terms), while in the improper model with $\mu_{Y}=0.0$, the investor considers a constant exposure to be optimal, and he ends up with an exposure to jump risk that is actually decreasing in the planning horizon (in absolute terms).

Figure 7 shows the results for the opposite case when the true model is without jumps in volatility. The true model is thus given by Parametrization III from Table 2, while the investor uses Parametrization I from Table 2. The realized exposures to the risk factors are increasing in absolute value compared to the optimal case, so that the investor holds positions with a higher level of risk. Whereas the optimal exposure to jump risk in the true model is now constant for all investment horizons, the realized exposure increases with the investment horizon in absolute value.

Knowledge of realized exposures under a mis-specified model is the necessary prerequisite for the determination of the utility loss suffered by an investor who bases his decision on an incorrect specification. The difference between optimal and realized risk exposures will in general depend on the differences in the parameters and risk premia as well as on the differences of the sensitivities of the traded assets under the true and the assumed model. This implies that the differences also depend on the current level of volatility. Due to this additional dependence on $V$, the indirect utility for the mis-specified model cannot be computed in closed-form as in Liu, Longstaff, and Pan (2003), who only consider trading in the stock and the money market account, i.e. in linear claims whose sensitivities cannot be mis-estimated. Instead, we have to determine the distribution of terminal wealth via a Monte Carlo simulation. Furthermore, the lower bound on the jump risk exposure which is supposed to prevent default can be imposed on the (seemingly) optimal exposure, but not on the realized exposure. Thus, default becomes possible in a mis-specified model. In the realistic case of $\gamma \geq 1$, the utility of terminal wealth will go 
to $-\infty$ when terminal wealth goes to zero. The indirect utility may thus take on large negative values, and the portfolio improvement as calculated in Section 5 has no real meaning anymore. We therefore focus on the comparison of the distributions of terminal wealth generated by the true and the mis-specified model.

In our simulation exercise we use 500,000 runs with two time steps per day. The traded assets available to the investor at every time step are the stock, the money market account, and two derivatives with a constant maturity of three months and strike prices equal to 90 and 100 percent of the current stock price. This implies that the investor trades in a new set of options every day. We simulate the dynamics of the investor's wealth using the realized exposures to the risk factors. It is important to note that the restriction on $\theta_{t}^{* N}$ as stated in Equation (13) is only monitored for the (seemingly) optimal exposure, but not for the realized one. This implies that the realized exposure can very well be too large, so that default becomes a possibility. In this case or when the simulation produces a negative value for wealth, we replace it by some positive number close to zero.

An implicit assumption behind our procedure is that there is no learning on the part of investors, i.e. the model is calibrated only once at time $t=0$. This is analogous to the approach chosen by Liu, Longstaff, and Pan (2003). Given that jumps are infrequent events, the estimation of jumps is quite difficult, which justifies to ignore the impact of learning in a first approximation. Further research could analyze how much the investor would really profit from learning about these infrequent events.

The results of the Monte Carlo simulation are given in Figure 8. The upper (lower) graphs show the cumulative distribution functions of terminal wealth for an investment horizon of 1 year (5 years). In the two graphs in the left column the true model contains jumps in volatility, whereas in the right column $\mu_{Y}=0$ in the true model.

In general the use of a wrong model generates a higher probability of large positive levels of terminal wealth, but also a higher risk of obtaining levels of wealth close to zero. Surprisingly, in the case where the true model does not contain volatility jumps and where the investor thus uses a model that is too sophisticated, the difference between the two distributions is even more pronounced. Additionally, we observe a rather high shortfall probability of roughly $5 \%$ for an investment horizon of 5 years. Figure 7 can help to explain this seemingly strange result. It shows that when a model with jumps in volatility is used, the realized exposures to the risk factors are much higher than optimal. Especially for jump risk, the realized exposure under model risk is much too high, so that if a jump occurs, wealth may easily become negative.

In summary our results emphasize the importance of identifying the correct model, since there is no easy way to hedge against the impact of model mis-specification when it comes to portfolio planning. The use of a more simple model gives rise to a significant default probability. This shows that a too parsimonious model is not robust. On the other hand, a too sophisticated model may perform even worse. Additional risk factors which are not included in the true model may actually lead to quite severe errors. Neither the use of a (perhaps) too simple nor the use of a (perhaps) too sophisticated model thus offers a reliable protection against significant losses in utility due to model mis-specification. 


\section{Conclusion}

Jumps in volatility are a phenomenon recently discussed in the literature dealing with the stochastic properties of stock prices or option pricing. One of the main questions is in which situations and for which problems the inclusion of this further risk factor has a significant economic impact.

We discuss the impact of such volatility jumps on the optimal asset allocation in a continuous-time framework. We suggest a very general formulation of the joint dynamics of the stock price and its variance by employing a jump size distribution which is able to capture both simultaneous jumps in both processes as well as individual jumps in the stock price or the variance. Nevertheless, we retain market completeness by introducing a sufficient number of additional traded assets (derivatives).

The main result of our theoretical analysis is that compared to an economy with no jumps in volatility the demand for jump risk now also exhibits a hedging component in addition to the usual myopic part. The hedging demand against unfavorable changes in volatility is now split up between the two diffusion risk factors and the jump risk factor.

Besides this theoretical innovation we also assess the economic benefits generated by the availability of derivatives to investors. In our framework derivatives actually complete the market, so that an investor who has access to derivatives can achieve any desired exposure to the fundamental risk factors. Our results show that the gains from trading derivatives are indeed economically significant.

Since complicated option pricing models are sometimes hard to calibrate to market data we investigate the cases in which an investor uses a simplified model ignoring volatility jumps completely or under-estimating the jump size in volatility. We find that this model or estimation error has a significant impact on optimal exposures and portfolio compositions and thus represents an important risk factor when choosing the optimal portfolio strategy. The same is true when the investor uses a model that includes jumps in volatility while the true model does not. The additional risk factor may cause quite severe deviations between the truly and the seemingly optimal portfolios. These results

emphasize the importance of identifying the correct model and show that the question of whether there are jumps in volatility should not be ignored.

Further research could focus on a general equilibrium model where the market prices of risk are no longer given exogenously. These market prices of risk as well as the trading volume in derivatives would then depend on the heterogeneity of the investors, which may differ with respect to their risk aversion or with respect to their prior beliefs about the model. 


\section{A Properties of the Function $H(\tau)$}

Since $H(0)=0$, it follows from the differential equation (15) that

$$
H^{\prime}(0)=\frac{1-\gamma}{2 \gamma^{2}}\left[\left(\eta^{B 1}\right)^{2}+\left(\eta^{B 2}\right)^{2}\right]+\frac{1-\gamma}{\gamma} \lambda^{Q}-\frac{1}{\gamma} \lambda^{P}+\lambda^{Q} \sum_{j, k} q_{j k}\left[\left(\frac{p_{j k} \lambda^{P}}{q_{j k} \lambda^{Q}}\right)^{1 / \gamma}\right]
$$

The first term on the right hand side, containing the risk premia for the two diffusion risk factors, obviously shares the sign of $1-\gamma$. With $\lambda^{P}=\alpha \lambda^{Q}$ and $\alpha>0$ rewrite the remaining terms as

$$
\frac{1-\gamma}{\gamma} \lambda^{Q}-\frac{1}{\gamma} \alpha \lambda^{Q}+\lambda^{Q} \sum_{j, k} q_{j k}\left[\left(\frac{p_{j k}}{q_{j k}}\right)^{1 / \gamma}\right] \alpha^{1 / \gamma}
$$

With $\lambda^{Q}$ positive, this expression will be positive if and only if

$$
f(\alpha)=\sum_{j, k} q_{j k}\left[\left(\frac{p_{j k}}{q_{j k}}\right)^{1 / \gamma}\right] \alpha^{1 / \gamma}-\frac{1}{\gamma} \alpha+\frac{1}{\gamma}-1
$$

is positive. The function $f(\alpha)$ has a local extremum at

$$
\alpha^{*}=\left(\sum_{j, k} q_{j k}\left[\left(\frac{p_{j k}}{q_{j k}}\right)^{1 / \gamma}\right]\right)^{-\gamma /(1-\gamma)}
$$

with

$$
f\left(\alpha^{*}\right)=\left(\frac{1}{\gamma}-1\right)\left(1-\alpha^{*}\right)
$$

The second derivative with respect to $\alpha$ is positive for $\gamma<1$ and negative for $\gamma>1$. So for $\gamma<1$ there is a global minimum at $\alpha^{*}$, whereas for $\gamma>1$ there is a global maximum at $\alpha^{*}$. With Jensen's inequality and $\sum_{j, k} q_{j k}\left[\frac{p_{j k}}{q_{j k}}\right]=1$, one can first show that $\alpha^{*} \leq 1$ for all values of $\gamma$. The associated function values $f\left(\alpha^{*}\right)$ are then negative in the case $\gamma>1$ and positive for $\gamma<1$, so that $f(\alpha)$ is non-negative for $\gamma<1$ and non-positive for $\gamma>1$.

Assume now $\gamma>1$. Then, $H^{\prime}(0)<0$, and the function $H(\tau)$ moves into negative territory over the first infinitesimal step in $\tau$ direction. Since the derivative of $H$ is negative when $H$ is equal to zero, and $H$ is continuous in $\gamma$, the function can never cross the zero line. An analogous argument can be made in the case when $\gamma<1$. Problems that can arise for very small values of $\gamma$ where the investor is nearly risk-neutral are discussed in Kim and Omberg (1996). 


\section{References}

Bakshi, G., C. Cao, and Z. Chen, 1997, Empirical Performance of Alternative Option Pricing Models, Journal of Finance 52, 2003-2049.

Bates, D.S., 1996, Jumps and Stochastic Volatility: Exchange Rate Processes Implicit in Deutsche Mark Options, Review of Financial Studies 9, 69-107.

Branger, N., and C. Schlag, 2004, Is Jump Risk Priced? - Properties of Tests Based on Option Hedging Errors, Working Paper.

Broadie, M., M. Chernov, and M. Johannes, 2005, Model Specification and Risk Premiums: Evidence From Futures Options, Working Paper.

Carr, P., and L. Wu, 2002, Static Hedging of Standard Options, Working Paper.

Daglish, Toby, 2002, The Economic Significance of Jump Diffusions: Portfolio Allocations, Working Paper.

Das, S., and R. Uppal, 2004, Systemic Risk and International Portfolio Choice, Journal of Finance 59, 2809-2834.

Das, S. R., and R. K. Sundaram, 1999, Of Smiles and Smirks: A Term Structure Perspective, Journal of Financial and Quantitative Analysis 34, 211-240.

Dieckmann, S., and M. Gallmeyer, 2004, The Equilibrium Allocation of Diffusive and Jump Risks with Heterogeneous Agents, Working Paper, forthcoming Journal of Economic Dynamics and Control.

Duffie, D., J. Pan, and K. Singleton, 2000, Transform Analysis and Asset Pricing for Affine Jump-Diffusions, Econometrica 68, 1343-1376.

Eraker, Bjørn, Michael Johannes, and Nicholas Polson, 2003, The Impact of Jumps in Volatility and Returns, Journal of Finance 58, 1269-1300.

Heston, S. L., 1993, A closed-form solution for options with stochastic volatility with applications to bond and currency options, Review of Financial Studies 6, 327-343.

Ilhan, A., M. Jonsson, and R. Sircar, 2004, Portfolio Optimization with Derivatives and Indifference Pricing, Working Paper.

Kim, T.S., and E. Omberg, 1996, Dynamic Nonmyopic Portfolio Behavior, Review of Financial Studies 9, 141-161.

Korn, R., and H. Kraft, 2004, On the Stability of Continuous-Time Portfolio Problems with Stochastic Opportunity Set, Mathematical Finance 14, 403-414.

Kraft, H., 2003, Optimal Portfolios and Heston's Stochastic Volatility Model, Working Paper. 
Liu, J., 2001, Dynamic Portfolio Choice and Risk Aversion, Working Paper.

Liu, J., F. A. Longstaff, and J. Pan, 2003, Dynamic Asset Allocation with Event Risk, Journal of Finance 58, 231-259.

Liu, J., and J. Pan, 2003, Dynamic Derivative Strategies, Journal of Financial Economics $69,401-430$.

Merton, R. C., 1971, Optimum Consumption and Portfolio Rules in a Contiuous-Time Model, Journal of Economic Theory 3, 373-413.

Merton, R. C., 1976, Option Pricing When Underlying Stock Returns are Discontinuous, Journal of Financial Economics 3, 125-144.

Munk, C., 2004, Dynamic Asset Allocation, Lecture Notes.

Munk, C., and C. Sørensen, 2004, Optimal Consumption and Investment Strategies with Stochastic Interest Rates, Journal of Banking and Finance 28, 1987-2013.

Wu, L., 2003, Jumps and Dynamic Asset Allocation, Review of Quantitative Finance and Accounting 20, 207-243. 


\begin{tabular}{|c|c|ccc|}
\hline & $Y=y^{(1)} \equiv 0$ & $Y=y^{(2)}$ & $\ldots$ & $Y=y^{(K)}$ \\
\hline$X=x^{(1)} \equiv 0$ & $p_{11}=0$ & $p_{12}$ & $\ldots$ & $p_{1 K}$ \\
\hline$X=x^{(2)}$ & $p_{21}$ & $p_{22}$ & $\ldots$ & $p_{2 K}$ \\
$\vdots$ & $\vdots$ & $\vdots$ & $\ddots$ & $\vdots$ \\
$X=x^{(J)}$ & $p_{J 1}$ & $p_{J 2}$ & $\ldots$ & $p_{J K}$ \\
\hline
\end{tabular}

Table 1: Jump Size Distribution

The table shows the structure of the jump size distribution in our model. $X$ is the random jump size in the stock price, $Y$ is the size of a variance jump, where we assume $Y \geq 0$. The event $X=0, Y=0$ is assigned a zero probability in the joint distribution of $X$ and $Y$, since it represents the event that no jump has occurred.

\begin{tabular}{|c|c|c|c|c|c|c|c|}
\hline & $\mu_{Y}$ & $\kappa^{P}$ & $\kappa^{Q}$ & $\bar{v}^{Q}$ & $\sigma_{V}$ & $\rho$ & $\eta^{B 2}$ \\
\hline I & 0.226 & 5.300 & 2.403 & 0.048 & 0.225 & -0.570 & -2.000 \\
II & 0.100 & 2.451 & 0.500 & 0.106 & 0.355 & -0.271 & -2.104 \\
III & - & 1.450 & 0.500 & 0.063 & 0.380 & -0.321 & -1.808 \\
\hline
\end{tabular}

Table 2: Calibrated Parameters

The table summarizes the results of the calibration. Parametrization I corresponds to the benchmark case of Liu, Longstaff, and Pan (2003). Parametrization III is the case without jumps in volatility, which corresponds to the model setup of Liu and Pan (2003). In Parametrization II, the variance jump size $\mu_{Y}$ is set equal to $0.1 \cdot \bar{v}^{P}=0.021721$, $\lambda^{P}=1.84156, \lambda^{Q}=11.64964, \mu_{X}=-0.25$ and $\eta^{B 1}=2.45112$ are the same for all parametrizations. 